Glasgow Caledonian

University

University for the Common Good

\title{
Scottish rheumatology sonography course: five-year experience of delivering a mentorship-model rheumatology ultrasound training programme accredited by the consortium for the accreditation of sonographic education
}

Ciechomska, Anna; McKay, Neil D.; Newcombe, Lisa; Brandon, Mhairi; Youssef, Hazem; Platt, Philip; Dale, James ; Dickson, Diane

Published in:

Scottish Medical Journal

DOI:

$10.1177 / 0036933020983569$

Publication date:

2021

Document Version

Author accepted manuscript

Link to publication in ResearchOnline

Citation for published version (Harvard):

Ciechomska, A, McKay, ND, Newcombe, L, Brandon, M, Youssef, H, Platt, P, Dale, J \& Dickson, D 2021,

'Scottish rheumatology sonography course: five-year experience of delivering a mentorship-model rheumatology ultrasound training programme accredited by the consortium for the accreditation of sonographic education', Scottish Medical Journal, vol. 66, no. 2, pp. 51-57. https://doi.org/10.1177/0036933020983569

\section{General rights}

Copyright and moral rights for the publications made accessible in the public portal are retained by the authors and/or other copyright owners and it is a condition of accessing publications that users recognise and abide by the legal requirements associated with these rights.

Take down policy

If you believe that this document breaches copyright please view our takedown policy at https://edshare.gcu.ac.uk/id/eprint/5179 for details of how to contact us. 


\section{Introduction}

In the past decades, interest in Musculoskeletal Ultrasound (MSUS) within the Rheumatology setting has steadily grown, and access to ultrasound equipment has become more widespread [1]. The emerging evidence base supports using point-of-care ultrasound to improve decision making and disease monitoring [2,3]. However, educational models supporting training in this area are diverse. There are a variety of ultrasound training methods available, ranging from online resources and local/national study workshops [4] to full competency based accredited programmes [5]. Ultrasound is recognised as a highly operator dependant tool, and guidance from national radiology societies outlines the necessity for formal training [6]. A survey of the Scottish Society for Rheumatology members in 2011 identified a lack of education/experience, and inadequate time available for training as the main barriers to performing rheumatology ultrasound [7].

The Scottish Rheumatology Ultrasound Group (SRUG) comprises of rheumatologists, rheumatology specialist nurses, podiatrists, physiotherapists and occupational therapists with a special interest in the use of ultrasound to assess rheumatic diseases, especially the inflammatory arthropathies. SRUG's mission is to develop the safe use of point-of-care ultrasound to improve rheumatology services. A priority issue is training. Consequently SRUG developed a faculty and mentoring network of rheumatology ultrasound experts with representation from the majority of health boards in Scotland (Greater Glasgow and Clyde, Lothian, Lanarkshire, Fife, Highlands, Grampian, Dumfries, Ayrshire and Borders) $[7,8]$. In 2014, in response to the lack of a standardised competency driven training route, NHS Education Scotland (NES) allocated financial support to SRUG who worked in partnership with Glasgow Caledonian University (GCU) to develop a focused ultrasound training course for health professionals working in the rheumatology setting. The outcome was the Consortium for the Accreditation of Sonographic Education (CASE) accredited Rheumatology Sonography Course (RSC).

\section{Methods}

\section{Course description}

The aim of the RSC is to ensure that rheumatology health professionals who undertake ultrasound are competent to practice and provide appropriate patient-centred care. The RSC is externally accredited by CASE, the organisation which accredits high quality sonographic courses delivered within the UK [5]. CASE accredited courses undergo revalidation every three years to confirm the ongoing quality of the course content and ensuring the course remains fit for purpose.

Joints of the hands and feet commonly demonstrate clinically relevant ultrasonographic findings and are frequently requested examinations within the rheumatology setting [9]. Furthermore, ultrasound examination has been consistently shown to be more sensitive than clinical examination at identifying inflammatory joint lesions (such as synovitis), especially in the feet $[10,11]$. Therefore, to develop competency in rheumatology ultrasound, in a short training window, the RSC curriculum is initially focused on detection of inflammatory pathology in the region of hands, wrists, ankles and feet. This focus was determined after discussion and review of service need by the RSC programme team and differs from previous UK training approaches which advocate a wider remit of anatomy and pathology 
including ultrasound guided aspiration and injection [12]. The Royal College of Radiology (RCR) advocates focused ultrasound training for non-radiology clinicians whose focused ultrasound skills enhance bedside assessment and help with treatment planning for patients [6].

Ultrasound practice under the supervision of an expert mentor is considered to be the most effective training method $[8,13]$. Yet, the limited number of mentors in some UK regions reduces the application of mentorship model in the nationwide ultrasound courses. By developing the network of mentors SRUG prepared the ground for the mentorship model of training which plays a paramount role in the RSC [7].

\section{Structure and delivery}

The RSC course supports professional development in focused ultrasound practice over a 12-month training period. A blended learning approach provides trainees with a face to face training workshop, online educational content and a programme of work-based ultrasound training under the supervision of an expert rheumatology mentor.

Course documentation and assessment guidance is provided, alongside four learning modules (Tab. 1) which are delivered through GCU's virtual learning environment (VLE), GCU Learn. The scope of theoretical knowledge is established by RCR [6] and required on all CASE accredited courses. Learning modules provide a learning platform with in-built assessments to test the learning prior to progression to the next learning module [14]. The educational materials use MRI images in planes corresponding to standard ultrasound planes to compare the appearance of anatomical structures demonstrated by these modalities and to place ultrasound image in a wider anatomical context. Radiograms are used to support learning of anatomy, help identify bone landmarks necessary for appropriate orientation of ultrasound probe, and to compare pathologies in case based learning.

Trainees are expected to engage with the online material in advance of a two-day training workshop. Throughout the workshop, trainees receive teaching about the use of ultrasound in the rheumatological setting, how to operate the ultrasound machine and optimise the image, standardised scanning techniques and recognition of normal and abnormal sonographic findings. The workshop is delivered by SRUG and external invited faculty including European Federation of Societies for Ultrasound in Medicine and Biology (EFSUMB) certified tutors. Two musculoskeletal sonographers and academics support the RSC directly, supervising the content of the on-line theoretical module and basic training in application of ultrasound in practise during workshop (DD and LN). To promote early acquisition of basic scanning skills the workshop provides trainees with extensive scanning time of healthy volunteers and rheumatology patients. Practical ultrasound sessions are complimented by keynote lectures that describe the role of ultrasound in specific clinical situations. This is followed by an interactive discussion between SRUG faculty members and trainees to allow learning to be applied across different professional backgrounds.

The workshop is followed by work-based ultrasound practice under the supervision of mentor to consolidate knowledge and develop ultrasound skills. Participants who do not have access to mentor in their workplace are assigned to mentor in the most convenient location. 


\section{Mentorship}

There are currently 13 active mentors supporting the RSC ( 8 within Scotland and 5 in England). Mentors for the RSC are approved by EFSUMB 2 Level criteria of practice (Tab.2) and attending SRUG/GCU mentor's development course. For each trainee, mentors provide regular clinical education, identify good practice or areas for improvement, facilitate staged assessments, appraise portfolio images, and confirm practical portfolio evidence. Mentors may also act as assessors at the Objective Structured Clinical Exam (OSCE).

Training is provided for mentors and OSCE assessors via a GCU online training package and a development workshop. The workshop includes expert lectures and guided scanning from recognised international leaders in ultrasound education. A seminar on the principles of mentoring from the GCU programme lead is also included. Mentors have the opportunity to attend the optional EFSUMB competency assessment session for professional development.

The mentorship role is key to trainee success, requires a high level of commitment and should be appropriately costed and funded [6]. SRUG is a community interest, not for profit registered business, and a forum for rheumatology ultrasound education and research. Funding for SRUG has been provided by a grant from NHS Education Scotland, RSC attendees' fees and pharmaceutical company sponsorship. Trainees who are unable to secure mentorship locally can pay an additional fee to be assigned a RSC mentor. Payment to RSC mentors is offered (at consultant hourly rate) to include hours per annum for supervising scanning and formative/staged assessment and feedback. A day rate is paid for faculty and assessors at the workshops and OSCE.

\section{Log of practical evidence}

Trainees maintain a portfolio which provides evidence of the developing clinical ultrasound skill and helps identify and resolve gaps in learning. This includes a record of clinical ultrasound hours (minimum 120 of which 50 must be supervised), anonymised image log (minimum 80 cases, including 50 normal scans and at least 10 abnormal cases for each anatomical region (hands, wrists, ankles and feet), reflections, case study, and formative assessment documentation.

\section{Formative and final competency assessments}

Central to clinical ultrasound progression is continuous formative assessment, which provides immediate feedback of the learning process to both the trainee and mentor. Formative assessment relates to both theoretical learning and ultrasound skills development. Engagement with the online educational material and multiple-choice questionnaires allow trainees to self-assess theoretical knowledge and understanding. Staged clinical assessments are performed during supervised scanning sessions with mentors. Staged assessment evaluates trainees' skill using the following criteria: preparation for scan, technical competence, patient care and communication, interpretation and reporting, critical evaluation of findings. Mentors document limitations, points of good practice and future action planning. Three formative staged assessments must be successfully completed prior to proceeding towards the final summative assessment. Trainees must complete their practical evidence portfolio, signed and verified by their clinical mentor before being eligible to attempt the final competency assessment.

To successfully complete the RSC, trainees must demonstrate achievement of all the learning outcomes (Tab. 1) during OSCE. The OSCE comprises of 4 stations which include assessment of scanning (normal 
and abnormal findings) and grading of synovitis activity. Each trainee rotates around the OSCE stations and is allowed 20 minutes per station to examine the subjects and present their findings to the assessor. The findings of the assessor are used as the reference for the trainee's findings [6].

The criteria within the OSCE assessment document were developed in line with CASE ultrasound competency criteria [5] and mirrors that of the staged formative assessments. On completion of all stations, the RSC assessment team review the OSCE assessment documents and determine if trainees are successful based on all criteria. Successful trainees are awarded the GCU CASE accredited 'Certificate of Competence to Practice Focused Rheumatology Ultrasound'. Unsuccessful trainees are provided with oral and written feedback, and following remedial action outlined within their feedback action plan, are eligible for a second and final assessment attempt.

After certification, participants are advised to undertake continuing professional development (CPD) which incorporates elements of ultrasound practice [6]. They are encouraged to continue building on their own portfolios by continuing to keep a log book of documented cases and CPD with reflections which should be included in annual appraisal and revalidation.

\section{Results}

Between 2014 and 2019 thirty-one trainees have enrolled onto the RSC course (Tab. 3). 22 trainees have successfully completed (71\%), three failed the OSCE (10\%; one passed the second attempt), two have withdrawn due to change of job role. 14 trainees (45\%) deferred OSCE for longer than 12 months (2 due to changing job role and loss of local mentor). Six participants (19\%) worked in England with the rest from Scottish Health Boards. At the beginning of 2020, there were 7 newly enrolled trainees and 7 deferred from previous year expected to sit the final OSCE by end of 2020. Due to the SARS-CoV-2 pandemic and the subsequent impact that this has had on trainees' access to practical training, assessment timelines have been deferred this year.

Trainees, mentors and assessors have evaluated the course positively with all trainees indicating clinical mentorship as the most positive, and course enrolment the least positive aspects of the course (Fig. 1) (Tab. 4).

Mentors all agreed that they received the information and support they needed to fulfil their role. One commented that their task was satisfying, but time-consuming, and that the remuneration offered was important when deciding to engage in the course.

\section{Discussion}

Competency assessment and certification in Rheumatology ultrasound is desirable to achieve a safe standard of practice within the UK. Mentorship is an important element of training, however, can be onerous and time consuming. To our knowledge, the RSC is the only CASE accredited course which offers financial recognition for the mentor's role and extends a mentor's ultrasound skill development, including optional EFSUMB certification. Rheumatology ultrasound is not a common skill and when the availability of regional mentors is limited, RSC mentors supervise trainees from external regions. 
The Canterbury Focused on Hands and Feet ultrasound course is another CASE accredited course designed for rheumatology practice [5]. It consists of two practice workshops and 400 hours of clinical ultrasound learning in the workplace in order to achieve the necessary clinical competencies [17]. In contrast to RSC, mentors are not financially recognised for their involvement in the Canterbury course and mentor supervision is dependent on goodwill [verbal communication].

Small cohorts of trainees in CASE accredited courses allow attentive, individualised training but it is time and cost intensive. We recommend that ultrasound mentorship should be recognised within the consultant rheumatologist's job role or remunerated accordingly. Rheumatology is yet to embrace ultrasound training in the specialist curriculum, partly due to the lack of widespread training capacity. Radiology services might be utilised instead of developing rheumatology ultrasound service, but this would be more demanding on radiologists, rheumatologists, and patients in terms of multiplying appointments.

The British Society for Rheumatology (BSR) recognises the need for ultrasound training by providing the Ultrasound for Rheumatology Practice (URP) [4]. The URP is a training programme consisting of online educational materials (free for BSR members) and practical workshops. Unlike CASE accredited courses URP does not offer assessment of competency. Participants still require embarking in regular ultrasound practice and should seek a mentor to supervise the progress of training.

Royal College of Radiology recommends that the duration of training in musculoskeletal ultrasound should be no less than 3 - 6 months with trainees performing approximately 10 supervised ultrasound examinations every week [6]. The scope envisaged for musculoskeletal ultrasound by RCR is much broader than in the RCS and includes other anatomical areas and pathologies rarely seen in rheumatology practice (e.g. injuries). Reduced exposure to scanning is sufficient to achieve competency in focused ultrasound technique. The end point of training programme is judged by an assessment of competencies. $10 \%$ of RSC candidates were unsuccessful in OSCE and required further support and additional scanning sessions.

Ultrasound examination can impact patient care significantly $[15,16]$, particularly in the early symptom presentation phase of inflammatory arthritis. Ultrasound scan of wrist, metacarpophalangeal and metatarsophalangeal joints improve on clinical predictive models for RA [15]. The RSC design facilitates trainees rapidly acquiring basic, clinically useful, ultrasound scanning skills (e.g. identification of synovitis) that can be built upon in a stepwise manner in later self-learning and accessory modular courses, promoted also by BSR URP. Training focused on scanning hands and feet is less demanding but still time consuming and difficult to fit in busy clinical environment. RSC expect completion within 12 months, yet extended training time was required for $45 \%$ of trainees because of work service pressure, changed job role or workplace. The trainees, and importantly the mentors, need to be motivated to complete the RSC at the time of enrolment and ideally within one placement.

The number of trainees who have completed the RSC is relatively small, yet they can ensure safe, focused ultrasound service in 11 different health boards. Participants on ultrasound courses that do not complete supervised ultrasound training are uncertain regarding safety of applying ultrasound to practice. Doctors are less likely to be supported by employers to provide ultrasound practice services unless assessed for competence. Radiology and rheumatology departments can work confidently together in provision of service when rheumatology adopts a minimum standard of competency for 
rheumatology ultrasound practice. The RSC is an example towards such a standard which, SRUG believe, is required in rheumatology ultrasound training.

\section{Acknowledgments}

RSC Mentor Group:

Nicola Alcorn, SRUG, NHS Grampian

Sandeep Bawa, SRUG, NHS Greater Glasgow and Clyde

Helen Harris, SRUG, NHS Lothian

John Harvie, SRUG, NHS Highlands

Lucy Moran, SRUG, NHS Dumfries and Galloway

Ismael Atchia, Northumbria Healthcare NHS Foundation Trust

Susan Earl, Royal Devon and Exeter NHS Foundation Trust

Nasimah Maricar, Manchester University NHS Foundation Trust

Ravik Mascarenhas, Royal Devon and Exeter NHS Foundation Trust

Ernest Wong, Portsmouth Hospitals NHS Trust

\section{Declaration of Conflicting Interests}

Dr Anna Ciechomska, Dr Neil McKay and Dr Mhairi Brandon received payment for ultrasound training on RSC course.

The author(s) disclosed receipt of the following financial support for the research, authorship, and/or publication of this article: This work was supported by NHS Education for Scotland, AbbVie Inc., Pfizer, MSD, Janssen, Eli Lilly, UCB, Biogen, Celgene, Sanofi

\section{References}

1. Mandl P, Ciechomska A, Terslev L, et al. Implementation and role of modern musculoskeletal imaging in rheumatological practice in member countries of EULAR. RMD Open. 2019;5(2):e000950. Published 2019 Jun 25. doi:10.1136/rmdopen-2019-000950

2. Colebatch AN, Edwards CJ, Østergaard M et al. EULAR recommendations for the use of imaging of the joints in the clinical management of rheumatoid arthritis. Ann Rheum Dis. 2013;72(6):804-14

3. Filippou G, Sakellariou G, Scire CA et al. The predictive role of ultrasound-detected tenosynovitis and joint synovitis for flare in patients with rheumatoid arthritis in stable remission. Results of an Italian multicentre study of the italian Society for Rheumatology Group for Ultrasound: the STAR. Ann Rheum Dis. 2018;77:1283-9.

4. British Society for Rheumatology. British Society for Rheumatology: Ultrasound for rheumatology practice. 2019. https://www.rheumatology.org.uk/ProfessionalDevelopment/Education-Events/Education-Courses/Ultrasound-for-rheumatology-practice

5. CASE. Consortium for the Accreditation of Sonographic Education. 2019. http://www.caseuk.org

6. The Royal College of Radiologists. BFCR(17)3 Ultrasound training recommendations for medical and surgical specialties, Third edition, 2017.

https://www.rcr.ac.uk/system/files/publication/field_publication_files/bfcr173_ultrasound_tra ining_med_surg.pdf

7. Ciechomska A, Dale J, McKay N et al. Lessons learned from the Scottish Rheumatology 
Ultrasound Mentoring Network project. Ann Rheum Dis. 72(Suppl 3):A151-A151

8. Taggart A, Alcorn N, Harris H et al. 259. Mobile Mentoring: An Ultrasound Training Initiative. Rheumatology. 2014;53(suppl_1):i156-i156.

9. Iagnocco A, Ceccarelli F, Perricone $\mathrm{C}$ et al. The use of musculoskeletal ultrasound in a rheumatology outpatient clinic. Med Ultrason. 2014;16(4):332-5.

10. Haavardsholm EA, Aga AB, Olsen IC et al. Ultrasound in management of rheumatoid arthritis: ARCTIC randomised controlled strategy trial. BMJ. 2016;354:i4205.

11. Karim Z, Wakefield RJ, Conaghan P et al. The impact of ultrasonography on diagnosis and management of patients with musculoskeletal conditions. Arthritis Rheum. 2001;44(12):29325.

12. Brown AK, Roberts TE, O'Connor PJ et al. The development of an evidence-based educational framework to facilitate the training of competent rheumatologist ultrasonographers. Rheumatology. 2007;46(3):391-7

13. Taggart AJ, Wright SA, Ball E et al. The Belfast musculoskeletal ultrasound course. Rheumatology. 2009;48(9):1073-6

14. Blackboard. Creating Learning Modules. 2019.

https://help.blackboard.com/Learn/Instructor/Course_Content/Create_Content/Create_Conta iners_for_Content/Create_Learning_Modules

15. Filer $\mathrm{A}$, de Pablo $\mathrm{P}$, Allen $\mathrm{G}$ et al. Utility of ultrasound joint counts in the prediction of rheumatoid arthritis in patients with very early synovitis. Ann Rheum Dis. 2011 Mar;70(3):500-7. doi: 10.1136/ard.2010.131573. Epub 2010 Nov 29. PubMed PMID: 21115552; PubMed Central PMCID: PMC3033529.

16. Horton SC, Tan AL, Wakefield R et al. Ultrasound-detectable grey scale synovitis predicts future fulfilment of the 2010 ACR/EULAR RA classification criteria in patients with new-onset undifferentiated arthritis. RMD Open. 2017;3(1):e000394.

17. Canterbury Christ Church University. Focused Ultrasound Course on Hands and Feet in Rheumatology. 2020. https://www.canterbury.ac.uk/medicine-health-and-socialcare/continuing-professional-development/focused-ultrasound-on-hands-wrists-and-feet-inrheumatology.aspx / 